\title{
Primär- und Sekundärprävention des Hirnschlags
}

Das Leitthema, das in mehreren Artikeln dieser Ausgabe behandelt wird, ist «Primär- und Sekundärprävention des Hirnschlags».

In der Schweiz erleiden jährlich ca. 16'000 Menschen einen Hirnschlag. Das Hirnschlagrisiko erhöht sich durch nicht beeinflussbare Risikofaktoren wie Alter und Veranlagung sowie durch behandelbare Risikofaktoren, welche durch den Lebensstil beeinflusst werden. Die zehn wichtigsten behandelbaren Risikofaktoren sind verantwortlich für rund $90 \%$ der Hirnschläge. Dies sind arterielle Hypertonie, Bewegungsmangel, Übergewicht, Dyslipidämie, Tabakkonsum, ungesunde Ernährung, kardiale Pathologien (wie z.B. Vorhofflimmern), Diabetes mellitus, psychosoziale Belastung/Stress/Depression und Alkoholüberkonsum [1].

Hirnschläge können sich körperlich auswirken, wie auch ein verändertes Fühlen, Verhalten und Denken verursachen. Hierdurch wird das Privat-, Arbeits- wie auch Sozialleben beeinflusst. Menschen, die bereits einen Hirnschlag erlitten haben, haben ein erhöhtes Risiko, einen zweiten Hirnschlag oder ein anderes vaskuläres Ereignis wie beispielsweise einen Herzinfarkt oder einen Verschluss einer Beinarterie zu erleiden. Ein Rezidivereignis erhöht das Risiko für Tod und Behinderung. Es lohnt sich, unmittelbar nach einem Erstereignis die Ursache bestmöglich abzuklären und vorbeugende Massnahmen zu initiieren respektive zu optimieren. Die Evidenzlage für einen Nutzen dieser Massnahmen ist hoch [2-4].

Die COVID-19-Pandemie unterstreicht die Wichtigkeit einer optimalen Primär- und Sekundärprävention. Vaskuläre Ereignisse, Erkrankungen und Risikofaktoren und COVID-19 sind stark miteinander verflochten. Es hat sich in COVID-19-Patienten ein erhöhtes Risiko von vaskulären Ereignissen gezeigt. Auch konnte eine höhere Rate an COVID-19-Infektionen sowie schweren Krankheitsverläufen mit schlechter Prognose in Patienten mit bekannten vaskulären Erkrankungen und Risikofaktoren gezeigt werden [5].

In den interessanten Beiträgen spannen national, ja sogar teilweise international anerkannte neurologische Experten den Bogen über alle Facetten des Leitthemas «Primär- und Sekundärprävention des Hirnschlags». Ge- winnen Sie einen Überblick über die aktuellen Erkenntnisse, Entwicklungen, Grenzen und Möglichkeiten der Primär- und Sekundärprävention des Hirnschlags. Ich wünsche Ihnen viel Freude beim Lesen dieser Artikel und hoffe, dass diese Sie im klinischen Alltag unterstützen und kritisches Denken und Diskussionen anregen. Ich verbleibe mit kollegialen Grüssen.

\section{Literatur}

1. O'Donnell MJ, Chin SL, Rangarajan S, Xavier D, Liu L, Zhang H, et al. Global and regional effects of potentially modifiable risk factors associated with acute stroke in 32 countries (INTERSTROKE): a case-control study. Lancet. 2016;388:761 - 5 .

2. Arnett DK, Blumenthal RS, Albert MA, Buroker AB, Goldberger ZD, Hahn EJ, et al. 2019 ACC / AHA guideline on the primary prevention of cardiovascular disease: a report of the American College of Cardiology/American Heart Association Task Force on Clinical Practice Guidelines. Circulation. 2019;140:e596 - 646.

3. Powers WJ, Rabinstein AA, Ackerson T, Adeoye OM, Bambakidis NC, Becker K, et al. Guidelines for the Early Management of Patients With Acute Ischemic Stroke: 2019 Update to the 2018 Guidelines for the Early Management of Acute Ischemic Stroke: A Guideline for Healthcare Professionals From the American Heart Association / American Stroke Association. Stroke. 2019;50:e344-418.

4. Willeit P, Toell T, Boehme C, Krebs S, Mayer L, Lang C, et al. STROKE-CARD care to prevent cardiovascular events and improve quality of life after acute ischaemic stroke or TIA: A randomised clinical trial. Lancet. 2020;25:100476.

5. Scutelnic A, Heldner MR. Vascular Events, Vascular Disease and Vascular Risk Factors-Strongly Intertwined with COVID-19. Curr Treat Options Neurol. 2020;22:40.

PD Dr. med. Mirjam R. Heldner

Universitätsklinik für Neurologie

Unversitätsspital und Universität Bern

Freiburgstrasse 10

3010 Bern

mirjam.heldner@insel.ch 